\title{
Benign Cystic Mesothelioma of the Peritoneum: A Rare Case and Review of the Literature
}

\author{
Safi Khuri ${ }^{\mathrm{a}}$ Hayim Gilshtein $^{\mathrm{a}}$ Wisam Abboud $^{\mathrm{a}}$ \\ Ahmad Assalia ${ }^{a, b}$ Yoram Kluger ${ }^{a, c}$ \\ ${ }^{a}$ Department of General Surgery, ${ }^{b}$ Division of Advanced Laparoscopy, and \\ 'Division of Surgical Oncology, Rambam Health Care Center, Haifa, Israel
}

\section{Key Words}

Mesothelioma · Cystic lesion · Peritoneum · HIPEC

\begin{abstract}
A 19-year-old male presented with right lower quadrant pain. Imaging studies revealed a cystic peritoneal mass. At surgery, a large peritoneal mass was excised. The pathology report revealed a benign cystic mesothelioma, and a right hemicolectomy with cytoreductive surgery was completed.
\end{abstract}

\section{Introduction}

Benign multicystic peritoneal mesothelioma was first described in 1979 by Mennemeyer and Smith [1]. It is a rare intra-abdominal tumor with a strong predilection for the surface of the pelvic viscera. Although considered by many to be benign [2], this tumor has a high local recurrence rate. When found in the peritoneal cavity, it is usually intimately attached to serosal surfaces of the intestine, omentum, spleen, liver or in the retroperitoneal space. The tumor has a female preponderance, with a female:male ratio of 5:1. Most patients have a history of a previous abdominopelvic operation, endometriosis or pelvic inflammatory disease. However, there are reports of the disease diagnosed in men [3] and children [4] as well as in rare extra-abdominal locations [5]. Many patients are asymptomatic and the tumor is found incidentally. Symptoms and signs in symptomatic patients are abdominal or pelvic pain and tenderness or a mass palpated on abdominal or pelvic physical exam. Clinical presentation depends on the size and location of the tumor, and can result, rarely, in dyspareunia, constipation and urinary symptoms. Controversy exists in regard to the pathogenesis of benign cystic peritoneal mesothelioma. While some believe this tumor is a neoplastic lesion due to its tendency to recur after surgical resection and the 
reported low incidence of previous abdominal infection, others [6] believe it is a reactive process. Some suggest a hormonal involvement in the tumor pathogenesis. The use of tamoxifen and long-acting GnRH analogues leads to a reduction in cyst volume and cyst growth [7]. Nevertheless, surgical excision with clear margins is the only curative option.

We describe a case of benign cystic mesothelioma treated in our department.

\section{Case Report}

A 19-year-old male presented to the Emergency Department complaining of right lower quadrant abdominal pain of 2 days' duration accompanied by diarrhea. The pain evolved gradually and did not radiate. His appetite was reduced but he suffered no vomiting. The patient's past medical history was unremarkable. His father had recently been treated for testicular lymphoma.

On physical examination upon admission, the patient's vital signs were normal. His temperature was $37^{\circ} \mathrm{C}$, and an abdominal examination revealed right lower abdominal quadrant tenderness. Neither guarding nor rebound was found. No abdominal mass was palpated. Rectal examination was normal. Complete blood count, urinalysis, liver and kidney function tests were all within normal limits. Abdominal ultrasound (US) was ordered for suspected appendicitis. This examination (fig. 1 , right panel) revealed a multicystic mass of $10 \times 3.4 \times 1.6 \mathrm{~cm}$ in the right iliac fossa. The appendix was not visualized. The patient complained of extreme pain upon compression of his abdominal wall with the US probe. Due to this finding, a computed tomography (CT) scan was ordered. Abdominal CT of the abdomen (fig. 1, left panel) revealed a thin-walled, cystic, multilobulated mass corresponding to the US finding. A small amount of fluid was noticed in the pelvis and surrounding the cystic mass. The appendix was normal.

Due to the cystic nature of the lesion, a cystic tumor was suspected. The patient was admitted to the surgical ward for further investigation. Chest CT and colonoscopy were unremarkable. CA 19-9 as well as CEA level were within normal limits. Subsequent diagnostic laparoscopy ( $\underline{\text { fig. } 2}$ ) revealed a grape-shaped pedunculated lesion, containing mucoid material (fig. 2, left panel). The entire lesion was twisted on a long stalk that originated from the cecal wall. The twist resulted in partial necrosis of the lesion. Multiple free intraperitoneal cystic lesions of various size were observed floating in the peritoneal cavity (fig. 2, middle and right panels). Other cystic lesions were noticed on the pelvic wall, peritoneal lining and around the liver (fig. 2, right panel). The mass and some of the free-floating cysts were carefully harvested and removed for histological evaluation using an endobag. Benign cystic mesothelioma was revealed in the pathology report. The patient recovered well from the operation and was readmitted after 6 weeks for additional treatment. He underwent cytoreductive surgery which resulted in complete removal of all cystic lesions. This necessitated right hemicolectomy due to the heavy load of multiple cystic lesions on the ascending colon wall. The patient recovered uneventfully.

\section{Discussion}

Diagnosis of benign cystic peritoneal mesothelioma is fraught with difficulties. In many patients, the diagnosis is made incidentally during investigation or surgery for other pathologies. Radiological tests including ultrasonography, CT and MRI may demonstrate the lesions but cannot differentiate them from other cystic lesions. Fine needle aspiration of the lesion is not informative. Exploratory laparoscopy is the most accurate diagnostic method since it allows local biopsy of the suspected tissue. Pathological differential diagnosis includes a number of benign (cystic lymphangioma, endometriosis and adenomatoid tumors) and malignant lesions (malignant mesothelioma and serous tumors involving the peritoneum). There are no evidence- 
based treatment strategies for benign cystic peritoneal mesothelioma. It is agreed that surgery is the only effective treatment, with complete removal of the cystic lesions as the mainstay of treatment and the only chance for avoiding local recurrence. An aggressive surgical approach including cytoreductive surgery with peritonectomy is therefore recommended. Malignant transformation of this benign tumor has been reported [8]. Some researchers [9] advocate aggressive surgery followed by heated intraperitoneal chemotherapy (HIPEC). The observation of malignant transformation mandates systematic clinical follow-up of these patients. Unfortunately, further followup is compounded by the fact that there are no reliable clinical or imaging features, or tumor markers. The prognosis is excellent. In one of the largest series reported in the literature, only 2 cases of death were reported [10].

\section{Conclusion}

Benign cystic peritoneal mesothelioma is a very rare tumor. It has a high recurrence rate with low malignant transformation rates. The mainstay of treatment is surgical eradication, with cytoreductive surgery and peritonectomy.
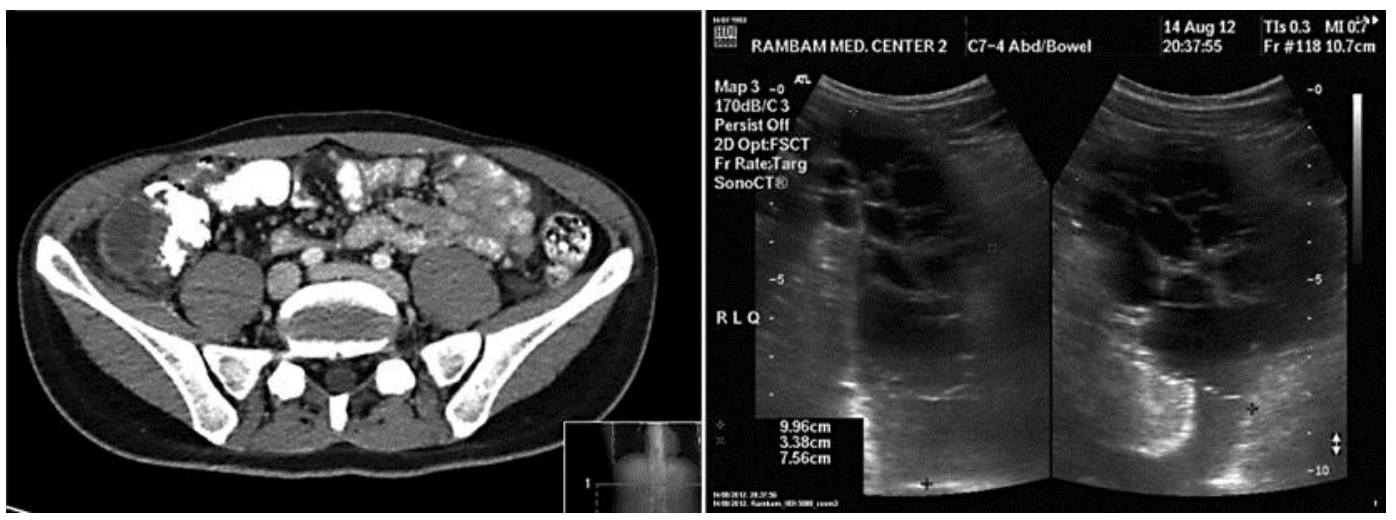

Fig. 1. US of the abdomen showing a multilobulated cystic mass in the right iliac fossa (right panel). CT scan of the abdomen revealed a multicystic, thin septated lesion (left panel). Free peritoneal fluid surrounds the cystic lesion. 

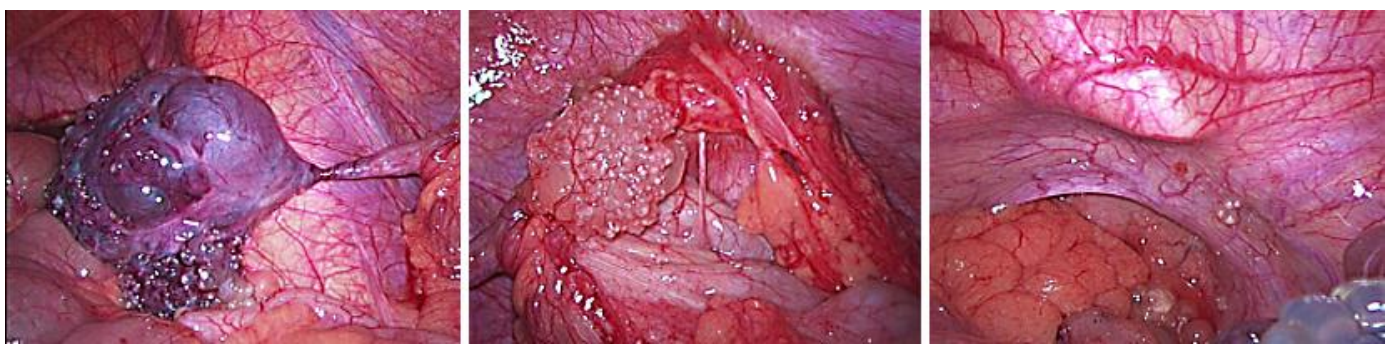

Fig. 2. Findings on diagnostic laparoscopy. A grape-shaped pedunculated lesion containing mucoid material (left panel). Multiple free intraperitoneal cystic lesions of various size were observed floating in the peritoneal cavity (middle and right panels).

\section{References}

1 Mennemeyer R, Smith M: Multicystic, peritoneal mesothelioma: a report with electron microscopy of a case mimicking intra-abdominal cystic hygroma (lymphangioma). Cancer 1979;44:692-698.

2 Van Ruth S, Bronkhorst MWGA, Van Coeverden F, et al: Peritoneal benign cystic mesothelioma: a case report and review of literature. Eur J Surg Oncol 2002;28:192-195.

-3 Machlenkin S, Diment J, Kashtan H: Benign mesothelioma of the peritoneum. IMAJ 2006;8:511-512.

-4 Shakya VC, Agrawal CS, Karki S, et al: Benign cystic mesothelioma of the peritoneum in a child-case report and review of the literature. J Pediatr Surg 2011;46:e23-e26.

-5 Ball NJ, Urbanski SJ, Green FH, Kieser T: Pleural multicystic mesothelial proliferation. The so-called multicystic mesothelioma. Am J Surg Pathol 1990;14:375-378

-6 Scucchi L, Mingazzini P, Di Stefano D, et al: Two cases of 'multicystic peritoneal mesothelioma': description and critical review of the literature. Anticancer Res 1994;14:715-720.

7 Letterie GS, Yon YL: The antiestrogen tamoxifen in the treatment of recurrent benign cystic mesothelioma. Gynecol Oncol 1998;70:131-133.

8 Gonzales-Moreno S, Jan H, Alcorn KW, et al: Malignant transformation of 'benign' cystic mesothelioma of the peritoneum. J Surg Oncol 2002;79:243-251.

-9 Sethna K, Mohamed F, Marchettini P, et al: Peritoneal cystic mesothelioma: a case series. Tumori 2003;89:31-35.

10 Weiss SW, Tavassoli FA: Multicystic mesothelioma. An analysis of pathologic findings and biologic behavior in 37 cases. Am J Surg Pathol 1988;12:737-746. 